\title{
Estrategia Neurodidáctica para la formación de investigadores sociales
}

\author{
Neurodidactic strategy for the training of social researchers \\ Estratégia neurodidáctica para a formação de investigadores sociais
}

DAVID OCAMPO EYZAGUIRRE ${ }^{1}$

\begin{abstract}
RESUMEN
El presente artículo realiza una descripción de los fundamentos teóricos y metodológicos de la Estrategia Neurodidáctica para la formación de investigadores sociales; misma, se desarrolló a partir de una revisión sistemática. El objetivo del presente estudio fue: desarrollar un análisis teórico sistematizada de las estrategias neurodidácticas; que permitan contribuir al mejoramiento del proceso de formación de investigadores sociales, como un proceso alternativo de formación de investigadores, que permita conocer, comprender, interpretar y transformar determinadas realidades y problemáticas sociales. La característica de este proceso fue: partir de la identificación de un conjunto de base de datos como: Redalyc, Scielo, Scopus, Latindex y Sciencie Academy, se desarrolló un análisis teórico; a partir, de una revisión sistematizada exploratoria. El plan de análisis de datos se realizó, utilizando el método Prisma: Identificación, selección, elegibilidad e inclusión; en la misma, los criterios de selección e inclusión para el análisis sistematizada del objeto de estudio fueron: El uso de estrategias neurodidácticas, estrategias para la formación de investigadores y escritos en español. Se presenta los fundamentos teóricos y metodológicos de una Estrategia Neurodidáctica, que permita contribuir a fortalecer la formación de investigadores nóveles en el área de las Ciencias Sociales.
\end{abstract}

Palabras clave: estrategia; neurodidáctica; ciencias sociales; investigadores sociales.

\begin{abstract}
The present article makes a description of the theoretical and methodological foundations of the Neurodidactic Strategy for the training of social researchers; it was developed from a systematic review. The objective of this study was to develop a systematized theoretical analysis of the neurodidactic strategies; that allow contributing to the improvement of the process of social researchers' formation, as an alternative process of researchers' formation, which allows knowing, understanding, interpreting and transforming certain realities and social problems. The characteristic of this process was from the identification of a set of databases such as: Redalyc, Scielo, Scopus, Latindex and Sciencie Academy, a theoretical analysis was developed; from a systematized exploratory review. The data analysis plan was carried out, using the Prisma method: identification, selection, eligibility and inclusion; in this way, the selection and inclusion criteria for the systematized analysis of the object
\end{abstract}


of study were: the use of neurodidactic strategies, strategies for the training of researchers and written in Spanish. It is presented the theoretical and methodological foundations of a Neurodidactic Strategy, which allows contributing to strengthen the formation of new researchers in the area of Social Sciences.

Keywords: strategy; neurodidactics; social sciences; social researchers

\section{RESUMO}

O presente artigo descreve os fundamentos teóricos e metodológicos da Estratégia Neurodidáctica para a formação de investigadores sociais; foi desenvolvido a partir de uma revisão sistemática. O objectivo do presente estudo foi: desenvolver uma análise teórica sistematizada das estratégias neurodidácticas; que permita contribuir para a melhoria do processo de formação de investigadores sociais, como um processo alternativo de formação de investigadores, que permita conhecer, compreender, interpretar e transformar certas realidades e problemas sociais. A característica deste processo foi: a partir da identificação de um conjunto de bases de dados tais como: Redalyc, Scielo, Scopus, Latindex e Sciencie Academy, foi desenvolvida uma análise teórica; a partir de uma revisão exploratória sistematizada. O plano de análise de dados foi realizado, utilizando o método Prism: identificação, selecção, elegibilidade e inclusão; nele, os critérios de selecção e inclusão para a análise sistematizada do objecto de estudo foram: a utilização de estratégias neurodidácticas, estratégias para a formação de investigadores e escritas em espanhol. São apresentados os fundamentos teóricos e metodológicos de uma Estratégia Neurodidáctica, que contribuirá para reforçar a formação de novos investigadores na área das Ciências Sociais.

Palavras-chave: estratégia; neurodidáctica; ciências sociais; investigadores sociais.

\section{INTRODUCCIÓN}

La formación de investigadores en el contexto de la Educación Superior, en la actualidad reviste una gran importancia, tomando en cuenta que el rol de las universidades es formar profesionales idóneos, capaces de transformar determinadas realidades en función de su objeto de estudio de cada ciencia y/o disciplina científica; en esa línea, el rol de las instituciones de Educación Superior, radica en la importancia de formar investigadores en diversas áreas del conocimiento científico, con un conjunto de destrezas de manejo teórico, metodológico y axiológico, expresados en un conjunto de habilidades y competencias investigativas.

De acuerdo a Vélez \& Villafuerte (2019) "una de las mayores dificultades, en la formación de investigadores se encuentra en la prevalencia del enfoque positivista arraigado en la academia del contexto latinoamericano..." (p. 45). Esta situación, pone en evidencia uno de los desafíos de la academia latinoamericana, diversificar los diferentes enfoques y paradigmas en el proceso de formación de investigadores sociales, privilegiando así las ideas, las motivaciones y la creatividad. 
En tal sentido, la formación de investigadores sociales reviste una importancia básica en los entornos académicos, fundamentalmente en el contexto de la Educación Superior, al respecto Padrón (2019) planteó la importancia y la necesidad de comprender y fortalecer los procesos de formación de investigadores al proponer que “... si no se comprende lo que es investigar, la estructura y su funcionalidad, no es posible investigar y mucho menos formar investigadores" (Carretero, 2019, p. 2). A partir de ello, es muy importante, desarrollar procesos de análisis respecto a la formación de investigadores sociales en el nivel de grado, dotando de habilidades, competencias teóricas y metodológicas, capaces de explicar e interpretar determinados problemas de la realidad social. Si se quiere enseñar a investigar, es decisivo cambiar el enfoque de la didáctica de la investigación científica, pasando de una enseñanza teórica, abstracta y general a una didáctica práctica, basada en la capacitación y el entrenamiento de las operaciones concretas que ocurren en la realidad, durante el proceso de la producción del conocimiento científico. El proceso de formación de investigadores en la Educación Superior, debe resultar de una relación entre la teoría y la práctica; es decir, no es basta la formación teórica, sino esto debe estar asociada a la práctica, aprender a investigar haciendo investigación.

La pregunta de investigación que ha guiado el presente estudio fue: ¿cómo contribuir a mejorar la formación de investigadores; a partir de la aplicación de estrategias neurodidácticas en el área de las Ciencias Sociales?, el objetivo de la investigación fue desarrollar un análisis teórico sistematizada de las estrategias neurodidácticas; que permitan contribuir al mejoramiento del proceso de formación de investigadores sociales. El argumento fundamental del presente estudio radica en la importancia de la inmersión de estrategias didácticas basadas en los fundamentos de la Neurodidáctica en el proceso de la formación de investigadores sociales cuyo fin es fortalecer la formación de investigadores nóveles en el área de las Ciencias Sociales.

\section{Fundamentos neurocientíficos de la neurodidáctica}

Los avances sobre Neurodidáctica están cada vez más presentes en la academia. Autores como Mora (2013); Marina (2019); Kandel et al. (1999); Ortíz (2009); Guillen (2017); solo para citar algunos de ellos, en los últimos 10 años han publicado numerosos estudios, que han contribuido a comprender la Neurodidáctica y sus fundamentos científicos. La Neurodidáctica es una disciplina científica reciente, que parte de la neurociencia y estudia la optimización del aprendizaje basado en el desarrollo del cerebro; sin duda, todo aprendizaje está basado en el funcionamiento del cerebro y las estructuras neuronales. En palabras de Ortiz (2009) "La Neurodidáctica está relacionado con la investigación del funcionamiento y la formación de micro-estructuras cerebrales, potenciadas esencialmente por estimulación para potenciar el desarrollo del aprendizaje".

A partir de las consideraciones planteadas, la Neurodidáctica es una disciplina pedagógica cuyo objeto de estudio son los procesos y elementos de aprendizaje con base en el funcionamiento del cerebro, que da una nueva orientación a la educación. Tiene como objetivo, diseñar estrategias didácticas y metodológicas eficientes, basados en el funcionamiento del cerebro. La Neurodidáctica, como disciplina que estudia la optimización del cerebro, tiene una estrecha relación con las neurociencias, que, en la actualidad, contribuyen a una mayor comprensión y en ocasiones a dar respuestas a cuestiones de interés para los docentes. 
Otro aspecto de mucha importancia a destacar es el rol que juega la experiencia, en la construcción de la estructura de la mente. El desarrollo cognoscitivo no es solamente un despliegue, de un conjunto de patrones pre-programados; hay convergencia en un conjunto de investigaciones sobre algunas de las reglas que gobiernan o dirigen el aprendizaje, una de las más simples, es que la "práctica incrementa el aprendizaje: en el cerebro, hay una relación similar entre la cantidad de experiencia en un ambiente complejo y el monto de cambio estructural" (Laura de la Barrera, 2009, p. 4).

En base a los aspectos mencionados, está claro que la práctica y la experiencia incrementa los procesos de aprendizaje, esto fortalece los cambios en los circuitos neuronales. Al respecto, Ocampo (2019) Plantea que "Durante el proceso de aprendizaje, los circuitos del cerebro sufren cambios. De su estudio se ocupa la neurodidáctica, una nueva disciplina según la cual la neurociencia puede ayudar a los educadores a desarrollar mejores estrategias didácticas" (p. 37).

Lo anterior hace ver, la importancia de desarrollar estrategias didácticas basada en los fundamentos de la Neurodidáctica, que permitan fortalecer los procesos de aprendizaje enseñanza; y en particular, contribuir a fortalecer procesos de formación de investigadores en el área de las Ciencias Sociales. A partir de ello, el aprendizaje tiene como base el sistema nervioso; el cerebro es el órgano que permite: pensar, desear, actuar, es el fundamento de múltiples y diferentes acciones conscientes como inconscientes permite responder a un conjunto de estímulos sean estímulos internos o externos.

En el proceso de formación de investigadores, es común encontrar diversos problemas, entre los que se destaca la falta de motivación. Es un tema que preocupa a estudiantes y profesores, por ello es importante desarrollar una estratégia neurodidáctica, que permita fortalecer los procesos de formación de investigadores en las Ciencias Sociales.

\section{Aproximaciones teóricas de las estrategias de enseñanza aprendizaje}

El proceso de aprendizaje y enseñanza en investigación; así como la formación de investigadores en las Ciencias Sociales es una tarea compleja, dado que intervienen muchos aspectos: sociales, psicológicos y culturales. En palabras de Carretero (2019; p.3) en el proceso de aprendizaje enseñanza de la investigación científica intervienen dos aspectos importantes: “ “. a) Lo que se enseña al enseñar a investigar y b) como se enseña a investigar. Estos dos tipos de operaciones constituyen dos prácticas distintas, la de producir conocimiento nuevo y la de enseñar a producirlo..."

En base a lo mencionado, formar investigadores en Ciencias Sociales, es una tarea que necesariamente requiere fortalecer determinadas habilidades y competencias en los estudiantes como en el docente, como elementos importantes del proceso docente educativo. Del mismo modo, la formación de investigadores está relacionado a generar y desarrollar un conjunto de habilidades y competencias investigativas. Es así que: "las estrategias didácticas [...] son los instrumentos de los que se vale el docente para contribuir a la implementación y el desarrollo de las competencias" (Pimienta, 2012, p. 3).

Por su parte, Díaz Barriga \& Hernández (2010) define la estrategia didáctica como: "los procedimientos que el agente de enseñanza utiliza en forma reflexiva y flexible para promover el logro de los aprendizajes significativos [...] son medios o recursos para prestar ayuda 
pedagógica" (p. 141). Se trata de un proceso que contribuye a la construcción y acompañamiento de aprendizajes. A partir de las consideraciones planteadas, la formación de investigadores y el aprendizaje y la enseñanza de la investigación científica en las Ciencias Sociales, está dirigido a fortalecer las habilidades y las competencias investigativas; además, de fortalecer el pensamiento crítico, profundizando las habilidades de toma de decisiones, en base al pensamiento crítico y argumentaciones, estructuradas de manera adecuada, que permita generar y sistematizar nuevos conocimientos en el área de las Ciencias Sociales.

\section{Estrategias neurodidácticas y sus fundamentos teóricos y metodológicos}

La neurociencia es considerada como un campo multidisciplinario, que investiga todo lo relacionado al cerebro y el sistema nervioso del ser humano (constitución, funcionamiento, manifestación, su desarrollo, etc.). En ese contexto, la naturaleza interdisciplinaria de la neurociencia permite la posibilidad de comprender en forma integral, el comportamiento humano. Según Tacca Huamán \& Alva Rodriguez (2019) "para una adecuada práctica docente, es muy importante conocer, el funcionamiento bioquímico del cerebro, ya que estos procesos son los responsables de la construcción del conocimiento humano" (p. 3). Entonces, el encargado de adaptar y ejecutar estas estrategias en él es el docente de acuerdo a las características del estudiante y el contexto donde se desarrolla el proceso docente educativo.

Siguiendo a Tacca Huamán \& Alva Rodriguez (2019, p. 18), las estrategias neurodidácticas se clasifican en: operativas, socio-emocionales y metodológicas: Las estrategias operativas representan el conjunto de estímulos creativos que planifica el docente para desarrollar un determinado contenido, responde al interés del estudiante y a las características del contexto. Las estrategias socio-emocionales involucran componentes emocionales que establecen los vínculos entre el docente - estudiante y las relaciones entre los estudiantes o pares, estas estrategias permiten el fortalecimiento del compromiso por el aprendizaje y la experiencia activa. Las estrategias metodológicas están compuestas por un conjunto de procedimientos que promueven la indagación, análisis y construcción del conocimiento, mediante procesos lógicos y con el apoyo de estratégias operativas y socioemocionales.

En base a los elementos mencionados, la utilización de las estrategias neurodidácticas en el proceso de formación de investigadores sociales, involucra prestar atención a cómo aprenden; es decir, los estilos de aprendizaje, al modo particular en que un estudiante se apropia de una determinada información; sin duda, en este proceso de formación de investigadores en el área de las Ciencias Sociales, las emociones constituyen un aspecto muy importante. Está demostrado que las emociones positivas estimulan la producción y liberación de dopamina, neurotransmisor que participa en diversos procesos cerebrales, cognitivos y emocionales. Según Elizondo et al. (2018) Esto significa, que, si un estudiante disfruta o experimenta situaciones agradables, las funciones cognitivas y los aprendizajes se incrementan sustantivamente.

A diferencia de la didáctica tradicional, las estrategias neurodidácticas desarrollan nuevas experiencias en el proceso aprendizaje y enseñanza de la investigación científica y por ende en la formación de los investigadores noveles en el área de las Ciencias Sociales, consideran el desarrollo cerebral y las necesidades de aprendizaje de los estudiantes, permitiendo disfrutar la experiencia de aprender para la vida. 


\section{METODOLOGÍA}

La presente investigación es el resultado de una revisión sistemática de tipo descriptivo, incluyendo en la investigación estudios cualitativos y cuantitativos. Al inicio del estudio se establecieron categorías de revisión, con las cuales se realizó el levantamiento de la información de la literatura seleccionada; a partir de ello, se realizó una sistematización de los resultados obtenidos.

En el proceso de la investigación, la búsqueda de la literatura fue realizada entre los meses enero, febrero, marzo de 2020, mismas fueron identificadas a partir de los siguientes bases de datos: Redalyc, Scielo; Scopus; Latindex y Sciencie Academy. Los términos de búsqueda o palabras claves en español fueron: "Estrategias neurodidácticas", "neurodidáctica", "neuroeducación", "estrategias didácticas", "didáctica", "neurociencias", "ciencias sociales", "investigadores sociales".

El proceso de revisión sistemática se desarrolló siguiendo los cuatro pasos del método PRISMA: Identificación, selección, elegibilidad e inclusión. Se consideró en el proceso la revisión de artículos a partir de los siguientes criterios de inclusión: Estudios que hicieran referencia a la neurodidáctica, estratégias neurodidácticas, estratégias didácticas en la formación de investigadores, estudios publicados entre el periodo 2014 y 2019, literatura escrita en español, escritos y artículos publicados en revistas indexadas en las bases de datos mencionadas con el propósito de garantizar la calidad de las publicaciones. Como criterios de exclusión se tomaron en cuenta los siguientes: artículos teóricos, meta análisis, ensayos y monografías; del mismo modo, se excluyeron del presente estudio publicaciones no indexadas o vagamente relacionadas con los criterios de inclusión.

Finalmente, en el proceso de la revisión sistemática se utilizó una base de datos construida para el proceso de análisis temático de los artículos seleccionado, definiéndose para ello, categorías iniciales, que se describen en la Tabla no. 1

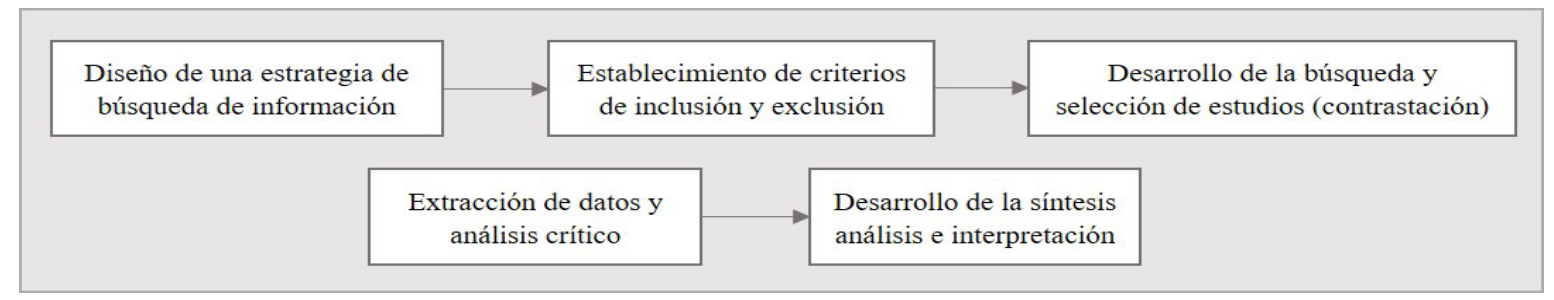

Figura 1. Proceso de revisión sistemática 
Tabla 1.

Definición de categorías de análisis

\begin{tabular}{|c|c|}
\hline Categoría & Definición \\
\hline Año & Fecha de publicación \\
\hline Revista & Revista de origen de publicación de artículo \\
\hline Metodología & Estudios cualitativos, cuantitativos y mixtos \\
\hline $\begin{array}{l}\text { Estrategias didácticas } \\
\text { para la formación de } \\
\text { investigadores }\end{array}$ & $\begin{array}{l}\text { Procedimientos, técnicas dirigidas a optimizar el proceso de } \\
\text { aprendizaje y enseñanza de los procesos de la investigación } \\
\text { científica. }\end{array}$ \\
\hline $\begin{array}{l}\text { Estrategias neuro di- } \\
\text { dácticas }\end{array}$ & $\begin{array}{l}\text { Procedimientos, técnicas de enseñanza aprendizaje, basados en } \\
\text { el funcionamiento del cerebro. }\end{array}$ \\
\hline Neurodidáctica & $\begin{array}{l}\text { Disciplina pedagógica que busca la optimización de los proce- } \\
\text { sos enseñanza aprendizaje en base al funcionamiento del cere- } \\
\text { bro. Como herramienta principal utiliza las emociones para que } \\
\text { el aprendizaje sea más efectivo. }\end{array}$ \\
\hline Neurociencias & $\begin{array}{l}\text { Campo de la ciencia que estudia el sistema nervioso, sus ele- } \\
\text { mentos como: su estructura, funciones y desarrollo a partir de } \\
\text { diversas concepciones: Psicológico, neurológico, biológico y } \\
\text { social. }\end{array}$ \\
\hline Ciencias sociales & $\begin{array}{l}\text { Ciencias que estudian el comportamiento del ser humano, en la } \\
\text { sociedad y sus formas de organización. }\end{array}$ \\
\hline
\end{tabular}

\section{RESULTADOS}

Se procedió analizar y sistematizar la información tanto a nivel descriptivo y cualitativo, lo que permitió desarrollar comparaciones e interpretaciones necesarias para el presente trabajo, cuyos resultados se presentan a continuación. La sistematización y procesamiento de los resultados se realizó utilizando el software de procesamiento de datos cualitativos ATLAS Ti Versión 7: Los resultados siguientes, evidencian las estratégias neurodidácticas posibles a ser utilizados en el proceso de formación de investigadores sociales.

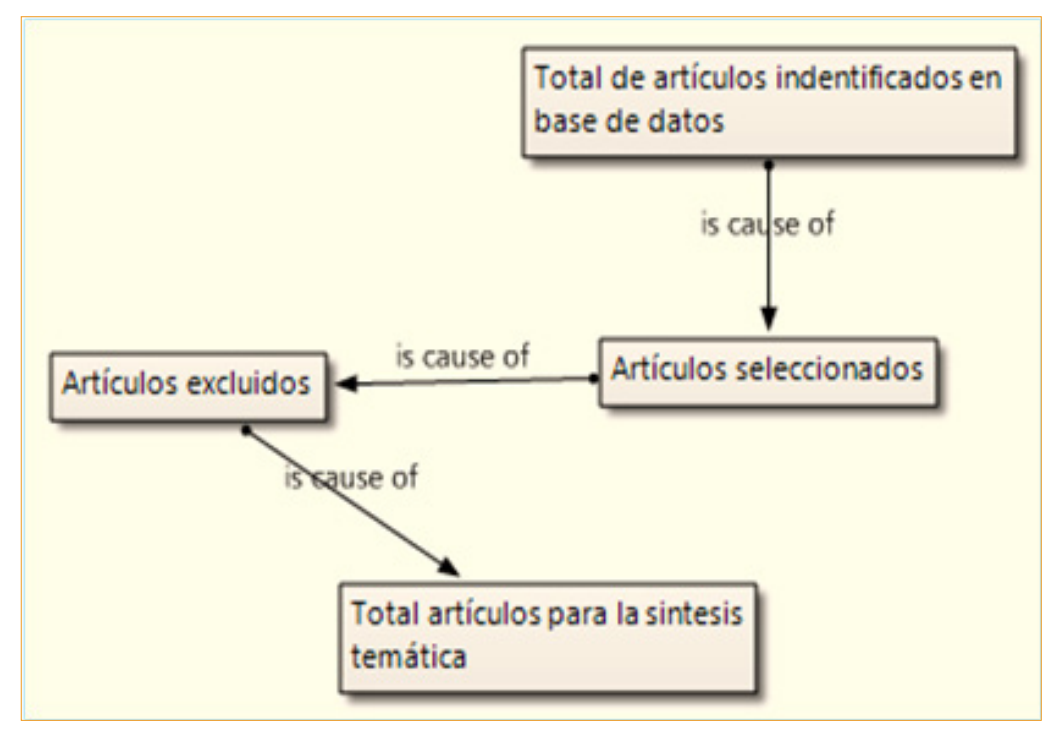

Figura 2. Resultados del proceso de búsqueda y selección de artículos 
El conocimiento científico respecto a las estrategias neurodidácticas para la formación de investigadores sociales es relativamente escasa a la actualidad. De acuerdo a la figura 2, el total de artículos identificados en las diferentes bases de datos, se han seleccionado en base al objeto de estudio a 117 artículos, 97 han sido excluidos en función a los diferentes criterios de selección propuestas para la presente investigación, un total de 20 artículos se eligieron para el desarrollo del estudio, los mismos que se presentan en la Tabla 2.

\section{Tabla 2.}

\section{Artículos seleccionados para la síntesis temática}

\begin{tabular}{|c|c|c|c|}
\hline Autores & Año & Nombre de publicación & Revista \\
\hline Pherez et al. & 2018 & $\begin{array}{l}\text { Neuroaprendizaje, una propuesta educativa: herramien- } \\
\text { tas para mejorar la praxis docente }\end{array}$ & $\begin{array}{l}\text { Civilizar, Ciencias Sociales y } \\
\text { Humanas }\end{array}$ \\
\hline Bueno \& Forés & 2018 & Neurodidáctica en el aula: transformando la educación & $\begin{array}{l}\text { Revista Iberoamericana de } \\
\text { Educación }\end{array}$ \\
\hline Carretero & 2019 & $\begin{array}{l}\text { Didáctica para la formación de investigadores, desde la } \\
\text { investigación vivencialista }\end{array}$ & $\begin{array}{l}\text { Revista. DELECTUS - Revis- } \\
\text { ta científica INIC-Perú }\end{array}$ \\
\hline Montenegro & 2017 & Didáctica universitaria de la investigación científica & $\begin{array}{l}\text { Revista Hacedor, versión elec- } \\
\text { trónica - Perú }\end{array}$ \\
\hline $\begin{array}{l}\text { Tacca Huamán \& } \\
\text { Alva Rodriguez }\end{array}$ & 2019 & $\begin{array}{l}\text { Estrategias neurodidácticas, satisfacción y rendimiento } \\
\text { académico en estudiantes universitarios }\end{array}$ & $\begin{array}{l}\text { Revista, Cuadernos de investi- } \\
\text { gación educativa }\end{array}$ \\
\hline Ferrer, K. y et al & 2020 & $\begin{array}{l}\text { Influencia de la neuroeducación en el rendimiento aca- } \\
\text { démico de estudiantes universitarios del área química }\end{array}$ & $\begin{array}{l}\text { EDUCERE. Revista venezola- } \\
\text { na de educación }\end{array}$ \\
\hline Rojas \& Aguirre & 2015 & $\begin{array}{l}\text { La formación investigativa en la Educación Superior } \\
\text { en América Latina y el Caribe: Una aproximación: Una } \\
\text { aproximación a su estado del arte }\end{array}$ & Revista Eleuthera \\
\hline $\begin{array}{l}\text { López de Parra } \\
\text { et al. }\end{array}$ & 2019 & $\begin{array}{l}\text { Representaciones sociales sobre prácticas investigativas. } \\
\text { Condiciones en la Universidad }\end{array}$ & $\begin{array}{l}\text { Revista Entramado, Universi- } \\
\text { dad libre de Cali }\end{array}$ \\
\hline $\begin{array}{l}\text { Arcila-Calderón } \\
\text { et al. }\end{array}$ & 2019 & $\begin{array}{l}\text { La formación del investigador social en la Universidad } \\
\text { Nacional de Colombia; Facultad de Ciencias Humanas }\end{array}$ & $\begin{array}{l}\text { Revista colombiana de socio- } \\
\text { logía }\end{array}$ \\
\hline $\begin{array}{l}\text { Casimiro Urcos } \\
\text { et al. }\end{array}$ & 2019 & $\begin{array}{l}\text { Estrategias didácticas para la enseñanza de la investiga- } \\
\text { ción científica }\end{array}$ & $\begin{array}{l}\text { Revista de ciencias humanas y } \\
\text { sociales }\end{array}$ \\
\hline Calatayud & 2018 & $\begin{array}{l}\text { Hacia una cultura neurodidáctica de la evaluación. La } \\
\text { percepción del alumnado universitario. }\end{array}$ & $\begin{array}{l}\text { Revista Iberoamericana de } \\
\text { educación }\end{array}$ \\
\hline Muchiut, et al. & 2018 & $\begin{array}{l}\text { Neurodidáctica y autorregulación del aprendizaje, un } \\
\text { camino de la teoría a la práctica }\end{array}$ & $\begin{array}{l}\text { Revista iberoamericana de } \\
\text { educación }\end{array}$ \\
\hline Paniagua & 2018 & Neurodidáctica: Una nueva forma de hacer educación & FIDES ET RATIO \\
\hline Valencia & 2014 & Pedagogía de las ciencias sociales & Revista de Estudios Sociales \\
\hline Rivera & 2015 & La interdisciplinariedad en las ciencias sociales & $\begin{array}{l}\text { Reflexiones, Universidad de } \\
\text { Costa Rica }\end{array}$ \\
\hline León & 2019 & Diálogos interdisciplinarios desde las Ciencias Sociales & $\begin{array}{l}\text { Estudios sobre las culturas } \\
\text { contemporáneas }\end{array}$ \\
\hline $\begin{array}{l}\text { Moreno-Peinado, } \\
\text { et al. }\end{array}$ & 2017 & $\begin{array}{l}\text { Estrategia didáctica para desarrollar el pensamiento } \\
\text { crítico }\end{array}$ & $\begin{array}{l}\text { REICE. Revista Iberoameri- } \\
\text { cana sobre calidad, Eficacia y } \\
\text { cambio en la Educación }\end{array}$ \\
\hline Bueno \& Torrens & 2017 & Neurociencia para educadores & ROSA SENSAT, Revista. \\
\hline Anton et al. & 2016 & $\begin{array}{l}\text { Neurodidáctica y estrategias de aprendizaje para la in- } \\
\text { clusión }\end{array}$ & $\begin{array}{l}\text { Revista nacional e internacio- } \\
\text { nal de educación inclusiva }\end{array}$ \\
\hline Navarro & 2018 & $\begin{array}{l}\text { Metodologías interdisciplinares como herramienta para } \\
\text { motivar }\end{array}$ & $\begin{array}{l}\text { Revista Iberoamericana de } \\
\text { educación }\end{array}$ \\
\hline
\end{tabular}




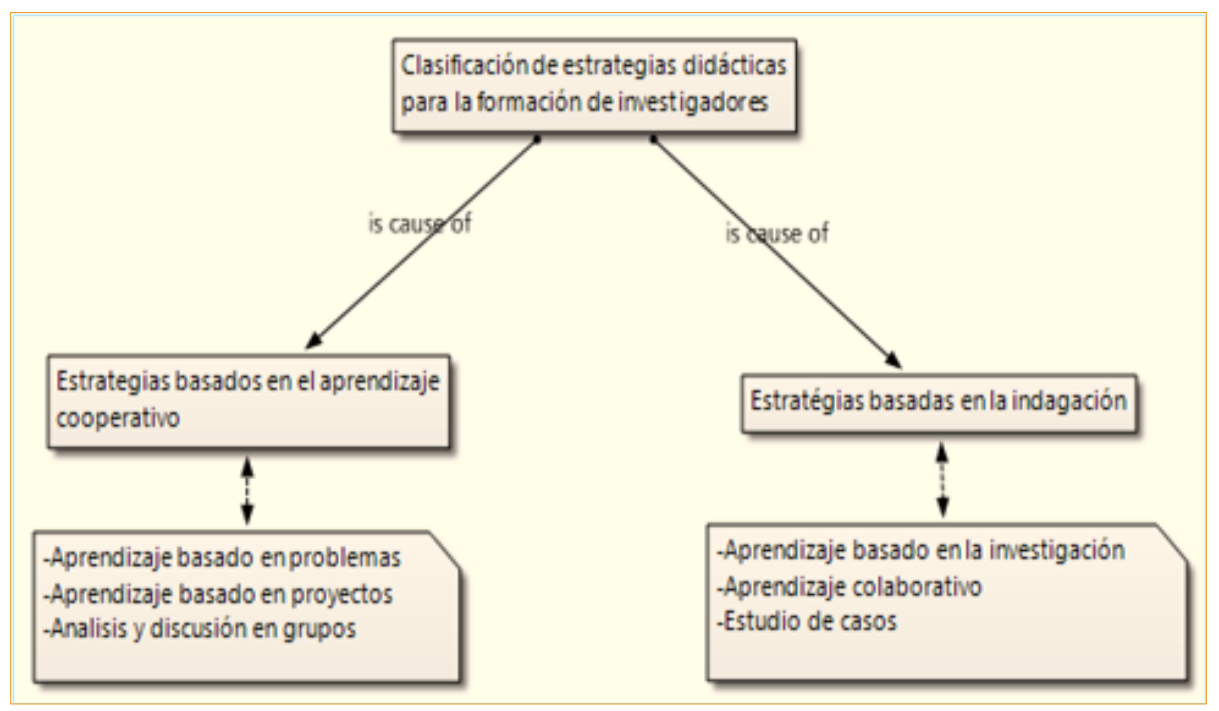

Figura 3. Clasificación de estrategias didácticas para la formación de investigadores

Las estrategias didácticas de aprendizaje y enseñanza se tratan de un conjunto de procedimientos, que utiliza el docente en el proceso de la implementación y desarrollo del proceso educativo, a fin de alcanzar los objetivos y/o competencias propuestas en cada uno de los niveles del proceso aprendizaje y enseñanza.

Montenegro (2017); Rojas \& Aguirre (2015); Casimiro Urcos et al. (2019), Arcila-Calderón et al. (2019) entre otros, han desarrollado estudios relacionados a las estrategias didácticas en la formación de investigadores; mismos coinciden en la necesidad de reforzar la didáctica de la investigación científica, fortaleciendo de esta manera la formación de investigadores en la Educación Superior. De la revisión sistemática realizada en el proceso de la investigación, se evidencia que el $60 \%$ de los artículos revisados plantean la importancia del aprendizaje cooperativo en el proceso de la enseñanza de la investigación científica; por otro lado, un $40 \%$ de los estudios revisados clasifican las estrategias didácticas en la formación de investigadores en: estrategias basados en el aprendizaje cooperativos y las estrategias basados en la indagación; Plantean la importancia de la formación de investigadores, a partir del aprendizaje cooperativo, que permita fortalecer la formación a partir del análisis de problemas, el aprendizaje basado en proyectos y el análisis y discusión en grupos. Por otro lado, el aprendizaje basado en la indagación se constituye en una alternativa que permita mejorar la formación de investigadores, el aprendizaje basado en la investigación, el aprendizaje colaborativo y el estudio de casos, se constituyen en una opción de estrategia didáctica en el proceso de formación de investigadores en el campo de las Ciencias Sociales. 


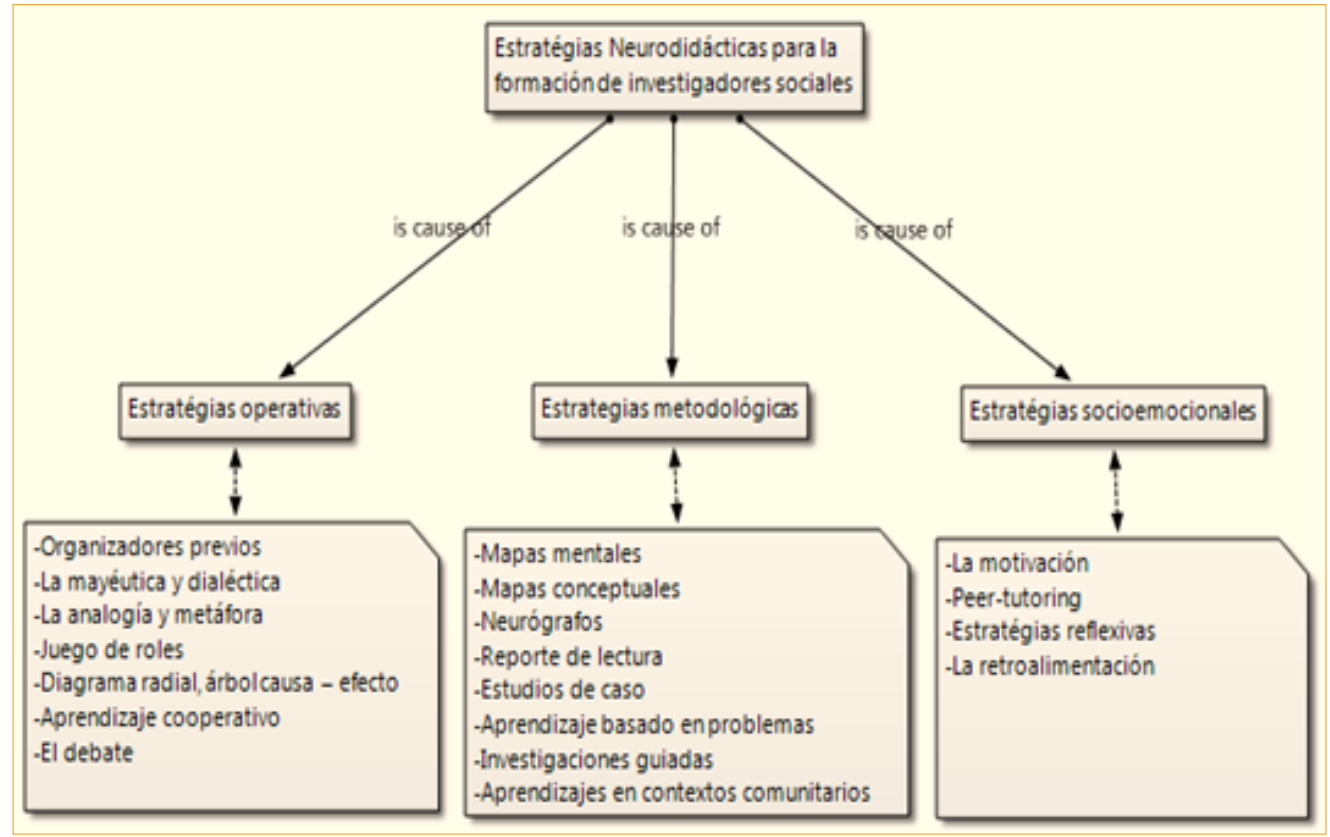

Figura 4. Estrategias Neurodidácticas para la formación de investigadores sociales

En relación a las estrategias neurodidácticas para la formación de investigadores sociales, de la revisión sistemática realizada en el proceso de la investigación, se evidencia que un $60 \%$ de los artículos revisados hacen notar la importancia de las emociones en el proceso de la formación de investigadores; del mismo modo, otros $60 \%$ de los artículos sujetos de revisión plantean la importancia del desarrollo de procesos de aprendizaje - enseñanza basados en el desarrollo del cerebro; es decir, la enseñanza basado en las características neuropsicológicas del estudiante. Para ello Pherez et al. (2018); Tacca Huamán \& Alva Rodriguez (2017); Bueno \& Forés (2018); Ferrer et al. (2020); Boscan (2011); entre otros, clasifican las estrategias Neurodidácticas en tres grupos: operativas, metodológicas y socioemocionales.

El proceso de formación de investigadores en el áreas de las ciencias sociales, debe ser de una manera integral (aspectos operativos, metodológicas y socioemocionales), las estrategias operativas, constituyen un conjunto de estilos creativos de enseñanza desarrollados en función al interés del estudiante, el contexto y las necesidades sociales; por otro lado, las estrategias metodológicas del proceso en cuestión, proporcionan procedimientos lógicos y metodológicos en la búsqueda y construcción del conocimiento, y las estrategias socioemocionales está dirigidos a fortalecer los procesos de interacción entre los aspectos fisiológicos, psicológicos y de carácter conductual en el proceso de formación de investigadores sociales. Se evidencia que cada cerebro es único y aprende de manera diferente en base a las características individuales, los aprendizajes en la investigación como en otras áreas son más eficaces cuando existe motivación; del mismo modo, se aprecia producto de la revisión sistemática la importancia del aprendizaje, desde, en y para la vida. 


\section{DISCUSIÓN}

La formación de investigadores en el área de las Ciencias Sociales, en la actual coyuntura constituye uno de los desafíos significativos de la Educación Superior; a partir de ello, es muy importante desarrollar investigaciones respecto a las estrategias aplicables al proceso de formación de investigadores sociales.

Las estrategias neurodidácticas en la formación de investigadores, se constituyen en una nueva forma de abordaje de la didáctica de la investigación científica basado en el funcionamiento del cerebro en base a las características neuropsicológicas de los estudiantes investigadores, especialmente nóveles, en el campo de las Ciencias Sociales. Se concuerda respecto a la importancia de realizar procesos de aprendizaje y enseñanza basados en estrategias neurodidácticas y el funcionamiento del cerebro (Mora, 2013; Ortíz (2018); Kandel (2019); Ramón Alonso (2018); Guillen (2017). En este sentido es importante poner énfasis en procesos de motivación, la estimulación y el aprendizaje cooperativo en los procesos de formación de investigadores sociales; de modo que los aprendizajes sean, desde en, y para la vida.

Los datos obtenidos, producto de la revisión sistemática en el presente estudio, permiten establecer procesos de reflexión académica; así como, un debate científico en torno a la utilización de una estrategia neurodidáctica en la formación de investigadores sociales.

La necesidad de formar investigadores en el área de las ciencias sociales, constituye un desafío de la educación superior; de modo, que permita fortalecer las competencias y habilidades de los estudiantes investigadores nóveles.

En relación a la categoría de las estrategias didácticas de formación de investigadores, se puede evidenciar que en las Ciencias Sociales las estrategias didácticas que se utilizan en la formación de investigadores se clasifican en dos grandes grupos: estrategias basadas en el aprendizaje cooperativo y estrategias basadas en la indagación. Las estrategias basadas en el aprendizaje cooperativo parten de la interacción con el entorno social; tales como: el aprendizaje basado en problemas, el aprendizaje basado en proyectos y el aprendizaje basado en el análisis y discusión de grupos, es importante hacer notar que estas estrategias promueven y fortalecen las habilidades y las competencias investigativas a partir de la relación con el entorno y el trabajo cooperativo. Las estrategias didácticas basados en la indagación, promueven las capacidades y habilidades investigativas de los estudiantes investigadores; a partir de, aprendizajes basados en la investigación y el estudio de casos.

En la categoría de la estrategia neurodidáctica para la formación de investigadores sociales se puede apreciar que autores como Ortíz (2018); Kandel (2019); Ramón Alonso (2018); Guillen (2017); Tacca Huamán \& Alva Rodriguez (2019) y Montenegro (2017); plantean la importancia de utilizar la estrategia neurodidáctica, como una alternativa, que permita fortalecer los procesos de formación de investigadores sociales. De la revisión teórica en la presente investigación se evidencia, la clasificación de la estrategia neurodidáctica en tres grupos: Estrategias operativas, las estrategias metodológicas y las estrategias socioemocionales. Esta clasificación plantea, que el proceso de formación de investigadores en Ciencias Sociales de una manera holística (integral), las estrategias operativas, constituyen un conjunto de estilos creativos de enseñanza desarrollados en función al interés y las caracterís- 
ticas socioculturales y psicológicas del estudiante, las estrategias metodológicas dirigidas a proporcionan procedimientos metodológicos de búsqueda y construcción del conocimiento y las estrategias socioemocionales dirigidas a fortalecer los procesos de interacción y cuestiones emocionales, enfatizando la motivación y los estímulos en los procesos de formación de investigadores sociales.

\section{CONCLUSIONES}

Posterior al desarrollo del análisis sistemático en la presente investigación, se presentan las conclusiones, considerando importante tener en cuenta las investigaciones relacionadas a la educación, basada en la Neurodidáctica, para el diseño de estratégias didácticas, que permitan fortalecer la formación de investigadores nóveles en el área de las Ciencias Sociales.

A la luz de los nuevos hallazgos en las Neurociencias, la estratégia Neurodidáctica constituye un referente muy importante en el proceso de la formación de investigadores en el área de las Ciencias Sociales; dado que se constituye en una nueva forma de abordaje de la didáctica de la investigación científica, basado en el funcionamiento del cerebro.

En el proceso de la revisión sistemática, se pudo evidenciar que existen una variedad de estratégias didácticas que son posibles de utilizar en la formación de investigadores sociales; entre los que sobresalen tenemos: las estrategias basadas en el aprendizaje cooperativo y las estrategias basados en la indagación, ambas clasificaciones, permiten fortalecer las habilidades y las competencias de los investigadores en su proceso de formación desde la práctica y la propia vivencia.

Las estrategias Neurodidácticas en el proceso de la formación de investigadores sociales, se constituyen en un conjunto de procedimientos basados en las características neuro-psicológicas de los estudiantes; mismas, ponen especial interés en las emociones y la importancia del proceso de aprendizaje y enseñanza basados en el desarrollo del cerebro; es así que, las estrategias Neurodidácticas en la formación de investigadores sociales, se clasifican en tres grupos: estrategias operativas, estratégias metodológicas y las estrategias socio-emocionales.

El proceso de formación de investigadores en las ciencias sociales, es concebido desde una manera holística (aspectos operativos, metodológicas y socioemocionales), desarrollando un aprendizaje, desde la vida (práctica), en y para la vida.

\section{REFERENCIAS}

Arcila-Calderón, C., Calderín-Cruz, M., \& Sánchez-Holgado, P. (2019). Adopción de redes sociales por revistas científicas de ciencias sociales. El profesional de la información, 28(1), 1-13. https://doi.org//10.3145/epi.2019.ene.05

Bueno, D. y Fóres A. (2018). 5 principios de la neuroeducación que la familia debería saber y poner en práctica. Revista Iberoamericana de Educación, 78(1), 13-25. https:// campus.autismodiario.com/wp-content/uploads/2019/02/282-75-PB.pdf\#page=7 
Carretero Valdez, S. V. (2019). Didáctica para la formación de investigadores desde la investigación vivencialista - intuitiva. DELECTUS - Revista cientifica, INICC-PE$R U^{\prime}, 2(2), 1-16$. https://doi.org/10.36996/delectus.v2i2.26

Casimiro Urcos, W. H., Casimiro Urcos, C. N., Barbachan Ruales, E. A., \& Ramos Ticlla, F. (2019). Estrategias didácticas para la enseñanza de la investigación científica. Oción, 2(89), 1470-1506. https://www.produccioncientificaluz.org/index.php/opcion/article/view/27532

Díaz Barriga, F., \& Hernández, G. (2010). Estrategias docentes para un aprendizaje significativo. Trillas.

Elizondo, A. Rodríguez, J. \& Rodríguez I. (2018) La importancia de la emoción en el aprendizaje. Didácticas específicas, (19), 37-42. https://repositorio.uam.es/bitstream/ handle/10486/686559/DE_19_3.pdf?sequence=1

Ferrer, K., Molero, L., Leal, A., Añez, O., Araque, M., \& Ávila, A. (2020). Influencia de la neuroeducación en el rendimiento académico de estudiantes universitarios del área de química. EDUCERE - Investigación arbitrada, 24(78), 223-236. http://erevistas. saber.ula.ve/index.php/educere/article/download/16034/21921927166

Guillen, J. (2017). Neuroeducación en el aula: De la teoría a la práctica. CreateSpace.

Kandel, E.R. (2019). La era del inconsciente. La exploración del inconsciente en el arte, la mente y el cerebro. Paidos.

Kandel, E.R. Schwartz, J.H., \& Jessell, T. M. (1999). Neurociencia y conducta. Editorial Prentice Hall

Laura de la Barrera, M. (2009). Neurociencias y su importancia en contextos de aprendizaje. Revista Digital Universitaria, 10 (4), 1-18. http://www.ru.tic.unam.mx:8080/tic/ bitstream/handle/123456789/1493/843.pdf?sequence=1\&isAllowed=y

Marina, J. A. (2019). Historia visual de la inteligencia. Conecta.

Montenegro, R. (2017). Didáctica universitaria de la investigación científica, valoración y propuesta desde la percepción de los estudiantes: Revista Hacedor, 1(2), 1-11. http:// revistas.uss.edu.pe/index.php/HACEDOR/article/view/744

Mora, F. (2013). Neuroeducación, solo se puede aprender aquello que se ama. Alianza.

Ocampo, D. (2018). Investigación cualitativa: Introducción a los fundamentos teóricos y metodológicos. Instituto Internacional de Investigación.

Ocampo, D. (2019). Neurodidáctica, Aportaciones al aprendizaje y la enseñanza. Instituto Internacional de investigación.

Ortíz, A. (2011). Psicopedagogía de las emociones. Sintesis

Ortíz, T. (2009) Aprendizaje y comportamiento basados en el funcionamiento de cerebro humano: Emociones, procesos cognitivos, pensamiento e inteligencia. Barranquilla.

Ortíz, T. (2018). Neurociencia en la escuela. Biblioteca de innovación educativa. 
Padrón, J. (2019). Teoría cognitiva de la investigación científica. Seminario I. Posdoctorado Internacional en Didáctica de la Investigación científica. Instituto Nacional de Investigación y Capacitación Continua - Perú.

Pherez, G., Vargas, S., \& Jerez, J. (2018). Neuroaprendizaje, una propuesta educativa: herramientas para mejorar la praxis del docente. Civilizar Ciencias Sociales y Humanas, 18(34), 149-166. http://www.scielo.org.co/pdf/ccso/v18n34/1657-8953ccso-18-34-00149.pdf

Pimienta, J. (2012). Estrategia de enseñanza aprendizaje: Docencia universitaria basada en competencias. Pearson.

Ramón Alonso, J. (2018) Mira al cielo. Editorial Juventud.

Rojas, C. \& Aguirre, S. (2015). La formación investigativa en la educación superior en América Latina y el Caribe: una aproximación a su estado del arte. Revista Eleuthera, 12, 197-222. https://doi.org/10.17151/eleu.2015.12.11

Tacca Huamán, D. R., \& Alva Rodriguez, M. A. (2019). Estrategias neurodidácticas, satisfacción y rendimiento académico en estudiantes universitarios. Cuadernos de Investigación Educativa, 10(2), 15-32. http://www.scielo.edu.uy/scielo.php?pid=S1688$93042019000200015 \&$ script $=$ sci_arttext\&tlng=en

Vélez, D. \& Villafuerte, R.W. (2019). Filosofia y didáctica en la formación de investigadores. Edit. Laripse. 